\title{
Stop taking routine biopsy specimens for the diagnose of a duodenal adenoma!
}

\section{(c) $(1) \ominus$}

\author{
Authors \\ Yasushi Yamasaki ${ }^{1}$, Noriya Uedo ${ }^{2}$ \\ Institutions \\ 1 Department of Gastroenterology, Okayama University \\ Hospital, Okayama, Japan \\ 2 Department of Gastrointestinal oncology, Osaka \\ International Cancer Institute, Osaka, Japan

\section{Bibliography} \\ Endoscopy International Open 2021; 09: E470-E471 \\ DOI 10.1055/a-1339-1089 \\ ISSN 2364-3722 \\ (c) 2021. The Author(s).
}

\begin{abstract}
This is an open access article published by Thieme under the terms of the Creative Commons Attribution-NonDerivative-NonCommercial License, permitting copying and reproduction so long as the original work is given appropriate credit. Contents may not be used for commecial purposes, or adapted, remixed, transformed or built upon. (https://creativecommons.org/licenses/by-nc-nd/4.0/)

Georg Thieme Verlag KG, Rüdigerstraße 14,

70469 Stuttgart, Germany
\end{abstract}

Corresponding author

Noriya Uedo, Department of Gastrointestinal Oncology, Osaka International Cancer Institute, 3-1-69 Otemae,

Chuo-ku, Osaka 541-8567, Japan

Fax: +81-6-6945-1902

noriya.uedo@oici.jp
Non-ampullary duodenal adenomas are relatively rare in the general population compared to other gastrointestinal neoplasms [1]; however, because they are considered precancerous, early detection and treatment are desirable [2,3]. Recently, because of advances in endoscopic technology, the opportunities for endoscopic detection and treatment of non-ampullary duodenal adenomas in clinical practice has increased. Endoscopic resection (ER) has been a first choice for treatment of non-ampullary duodenal adenomas, but the incidence of adverse events (AEs) such as immediate/delayed perforation and bleeding is not negligible [4]. Besides the conventional injection endoscopic mucosal resection (EMR) technique, several ER techniques, such as cold snare polypectomy (CSP), underwater endoscopic mucosal resection (UEMR), endoscopic submucosal dissection (ESD) with complete closure and laparoscopy-endoscopy cooperative surgery (LECS) have been developed to prevent AEs and are available [5].

Although the indication for each ER technique is not yet fully standardized, these techniques are applied based on lesion size and histological grade. Basically, CSP is used for small $(<10 \mathrm{~mm})$ low-grade adenoma, UEMR for small-to-intermediate $(<20 \mathrm{~mm})$ low- and high-grade adenoma, and ESD with complete closure or LECS for large (>20 mm) high-grade adenoma [5]. CSP, in particular, is extremely safe and a rate of AEs similar to biopsy [6], so it can be performed as excisional biopsy. ESD with complete closure has been performed in only expert of high-volume centers even in Japan.

To reduce the rate of AEs related to ER for superficial nonampullary duodenal epithelial tumors, optical endoscopic diagno- sis also is useful. Histological diagnosis with endoscopic biopsy has been the gold standard for clinical decision-making, but forceps biopsy induces fibrosis in the submucosa, makes subsequent ER difficult, and may increase the rate of AEs [7]. Kinoshita et al. [7] reported that in about $24 \%$ of duodenal adenomas in which injection EMR was initially attempted, removal was performed by conversion ESD because of the non-lifting sign caused by biopsy. In fact, the fibrosis caused by forceps biopsy makes even ESD difficult. Thus, as with colorectal adenomas, ER for non-ampullary adenomas without forceps biopsy would be a practical approach. The diagnostic accuracy of endoscopic forceps biopsy for final histology reportedly is not very high (sensitivity of $37.5 \%$ and specificity of $83.1 \%$ ) [7].

To determine the indication for and method of ER, differential diagnosis of the following pathological entities is important: non-neoplasia vs. neoplasia, and low-grade adenoma vs. high-grade adenoma/adenocarcinoma. In 2020, we reported that pit-type surface patterns in magnifying endoscopy with narrow band imaging (M-NBI) were useful for discriminating duodenal adenoma from non-neoplasia [8]. We found that this finding was not useful for lesions in the duodenal bulb because they showed various types of surface patterns (e. g. gastric type adenoma showed groove type patterns, gastric metaplasia showed pit type pattern, and Brunner's gland hyperplasia showed groove type pattern). However, for lesions in the second and third portion, we found that pit-type surface pattern had high diagnostic accuracy: sensitivity of $85 \%$, specificity of $93 \%$, positive predictive value of $97 \%$, and negative predictive value of $67 \%$, respectively [8]. Serious AEs related to ER usually 
develop in the post-ampullar location due to exposure of the resection wound to pancreatic juice and bile [4]. Therefore, we considered that endoscopic diagnosis based on this simple criterion could avoid unnecessary biopsy for confirmation of adenoma in post-ampullar lesions, and would reduce AEs of duodenal ER. Regarding differentiation between low- and high-grade adenoma, the usefulness of several diagnostic criteria in M-NBI have been reported [8-10]. Kikuchi. [9] proposed a diagnostic algorithm using surface and vascular pattern in M-NBI, and reported that a lesion with mixed surface pattern was suspicious for high-grade adenoma. Kakushima et al. [10] reported that a mixed or disappeared surface pattern in M-NBI was suggestive of high-grade adenoma, which was consistent with our study result [8]. Tsuji et al. adapted the vessel plus surface (VS) classification system, which was developed for diagnosis of early gastric cancer, to diagnosis of non-ampullary duodenal adenomas. They found that high-grade adenoma had an irregular microsurface pattern more frequently than low-grade adenoma; however, there was no difference in prevalence of irregular microvessels between low- and high-grade adenoma [11]. Each diagnostic criterion using M-NBI seemed useful to a certain degree, but they did not have diagnostic capability sufficient to totally replace forceps biopsy.

In this issue of Endoscopy International Open, Tsuji et al. [12] investigate the usefulness of the VS classification using M-NBI for differential diagnosis of low- and high-grade adenoma, taking into consideration the influence of previous biopsy. In the study by Tsuji et al., M-NBI images of adenomas were classified into two groups: a non-biopsy group in which forceps biopsy was not performed before M-NBI examination and a biopsy group in which forceps biopsy was performed before M-NBI examination. In the non-biopsy group, the accuracy rate for differentiation between low- adenoma and high-grade adenoma/adenocarcinoma was better than in the biopsy group ( $88 \%$ vs $66 \%, P=0.02)$. Notably, the specificity in the biopsy group was extremely lower than in the non-biopsy group (14\% vs. $70 \%, P<0.01)$, suggesting that most low-grade adenomas were over-diagnosed as high-grade adenoma/adenocarcinoma in the biopsy group [12]. Although the study had several limitations regarding small sample size and retrospective evaluation of the recorded images (two M-NBI experts reviewed endoscopic images), it suggests that the low diagnostic ability of M-NBI was, in part, caused by forceps biopsy.

Needless to say, so far, the current gold standard for clinical decision-making is the histology of biopsy specimens; however, it is important to inspect the lesion carefully with endoscopy, especially using image-enhanced endoscopy (IEE), before forceps biopsy. When the endoscopic diagnosis of adenoma was made with high confidence according to the reported diagnostic criteria [8-12], and drawbacks of forceps biopsy are presumed, ER without forceps biopsies is preferrable. In practice, biopsy could be performed for a large polypoid tumor, choosing the most suspicious area of neoplasia or high-grade histology. But for superficial lesions with reliable endoscopic findings, routine endoscopic forceps biopsies should be avoided.
Otherwise, if it is attempted, the number and size of biopsy specimens should be minimized by use of thin forceps or sampling from the lesion margins.

In the future, the endoscopic diagnostic criteria for non-ampullary duodenal adenomas will be unified and differentiation of neoplasia from non-neoplasia and high-from low-grade adenoma will be included. Development of safe and practical diagnostic and therapeutic strategies based on optical endoscopic diagnosis using M-NBI or other IEE is warranted.

\section{Competing interests}

The authors declare that they have no conflict of interest.

\section{References}

[1] Jepsen JM, Persson M, Jakobsen NO et al. Prospective study of prevalence and endoscopic and histopathologic characteristics of duodenal polyps in patients submitted to upper endoscopy. Scand J Gastroenterol 1994; 29: 483-487

[2] Sellner F. Investigations on the significance of the adenoma-carcinoma sequence in the small bowel. Cancer 1990; 66: 702-715

[3] Okada K, Fujisaki ], Kasuga A et al. Sporadic nonampullary duodenal adenoma in the natural history of duodenal cancer: a study of followup surveillance. Am J Gastroenterol 2011; 106: 357-364

[4] Inoue T, Uedo N, Yamashina T et al. Delayed perforation: a hazardous complication of endoscopic resection for non-ampullary duodenal neoplasm. Dig Endosc 2014; 26: 220-227

[5] Yamasaki Y, Uedo N, Takeuchi Y et al. Current status of endoscopic resection for superficial nonampullary duodenal epithelial tumors. Digestion 2018; 97: 45-51

[6] Hamada K, Takeuchi Y, Ishikawa H et al. Safety of cold snare polypectomy for duodenal adenomas in familial adenomatous polyposis: a prospective exploratory study. Endoscopy 2018; 50: 511-517

[7] Kinoshita S, Nishizawa T, Ochiai Y et al. Accuracy of biopsy for the preoperative diagnosis of superficial nonampullary duodenal adenocarcinoma. Gastrointest Endosc 2017; 86: 329-332

[8] Yamasaki Y, Takeuchi Y, Kanesaka T et al. Differentiation between duodenal neoplasms and non-neoplasms using magnifying narrowband imaging - Do we still need biopsies for duodenal lesions? Dig Endosc 2020; 32: 84-95

[9] Kikuchi D, Hoteya S, lizuka T et al. Diagnostic algorithm of magnifying endoscopy with narrow band imaging for superficial non-ampullary duodenal epithelial tumors. Dig Endosc 2014; 26: (Suppl. 02): 16-22

[10] Kakushima N, Yoshida M, Yamaguchi Y et al. Magnified endoscopy with narrow-band imaging for the differential diagnosis of superficial non-ampullary duodenal epithelial tumors. Scand J Gastroenterol 2019; 54: 128-134

[11] Tsuji S, Doyama H, Tsuji K et al. Preoperative endoscopic diagnosis of superficial non-ampullary duodenal epithelial tumors, including magnifying endoscopy. World J Gastroenterol 2015; 21: 1183211841

[12] Tsuji S, Doyama H, Tsuyama S et al. Does previous biopsy lead to cancer overdiagnosis of superficial non-ampullary duodenal epithelial tumors using magnifying endoscopy with NBI? Endosc Int Open 2020; 09: E58-E65 